\title{
Sclerosing Thymoma
}

National Cancer Institute

\section{Source}

National Cancer Institute. Sclerosing Thymoma. NCI Thesaurus. Code C45709.

A rare type of thymoma, characterized by an exuberant amount of collagen-rich stroma. 